\section{穴 Heighten Science \\ P U B L I C I T I O N S Corporation ISSN \\ 2573-7724}

\title{
Metallic Ureteric Stents a cost-effective alternative in the management of Obstructed Distal Ureters
}

\author{
Erich K Lang* \\ Professor Radiology \& Urology, Tulane School Medicine, USA
}

\footnotetext{
*Address for Correspondence: Erich K Lang Professor Radiology \& Urology, Tulane School Medicine, USA, Email:

Erich.Lang@downstate.edu

Submitted: 06 April 2017

Approved: 02 May 2017

Published: 04 May 2017

Copyright: $\circledast 2017$ Lang EK. This is an open access article distributed under the Creative Commons Attribution License, which permits unrestricted use, distribution, and reproduction in any medium, provided the original work is properly cited.
}

\section{INTRODUCTION}

Management of obstruction of the distal-pelvic ureters by minimally invasive procedures such as antigrade or retrograde double "J" stents has generally been unsatisfactory. Corrective surgical procedures mandate long hospitalization, hence high cost, and have a moderate incidence of complications.

\section{MATERIAL AND METHODS}

Twenty-five patients with advanced Carcinoma of the Cervix occluding the pelvic ureters, hence compromising renal function, which in turn curtailed adjunct management by chemotherapy, were managed by metallic wall stents to relieve the obstruction and restore renal function. Fourteen wallstents remained patent 18 months or longer, with creatinine levels maintained at 1.2 -1.8.

Because of tumor-ingrowth through non-covered wall stents, 56 endo-urologic salvage procedures were necessary to maintain patency.

In the last 10 years we have placed 22 thermo-expandable metallic stents for correction of 17 neoplastic and 5 benign ciccaticial obstructions of the ureter with similar success [1].

Obstruction of the distal-pelvic ureters by neoplastic or inflammatory disease poses vexing problems. Surgical interventions, such as reimplantation of the ureter with psoas hitch, Boari flap or ureteroneocystostomy will re-establish drainage of urine from the kidney [1-3]. Creation of an orthotopic sigmoid neobladder will likewise effectively correct the obstruction and prevent permanent damage to renal function [4]. However, although initial results with reimplantation tend to be satisfactory, the eGFR deteriorates significantly over time in male patients [2]. Orthotopic neobladders have an early complication-rate of $22.6 \%$ and a late complication-rate of $25 \%$ [4]. These surgical procedures are complex and attendant hospitalization time and cost are significant.

Management by antigrade or retrograde double "J" stents is generally unsatisfactory. Even if stent placement is accomplished after dilatation of the existing strictures functional life of the stents is short. Strictures at the uretero-vesical or ureterointestinal junction can be managed by Acucise balloon dilatation and subsequent seating of a double "J" stent. Successrates of $83 \%$ for uretero-vesical strictures and $50 \%$ for ureteroileal strictures have been reported [5].

The advent of metallic stents for treatment of obstruction of the distal ureters has introduced a relatively minimally invasive modality to manage the complications of ureteric obstruction by neoplasm, endometriosis, retroperitoneal fibrosis, and inflammatory or post-radiation therapy [6,7]. 
The collecting system is accessed via the interpolar calyx by percutaneous nephrostomy. Under fluoroscopic guidance an $\mathrm{H} 1$ or Bern curve catheter (Boston Scientific) is advanced to the site of obstruction in the distal ureter. A stiff Amplatz guide wire is forced into the obstructed segment and an Acucise balloon catheter used to incise and dilate the strictured area. Alternatively a laser incision along the stricture can be used. Following dilatation of the strictured segment a metallic stent (Wallstent or thermoexpandable metallic stent (manufactured by PNM Medical, Denmark) $t$ is seated over the guide wire, extending into the bladder-lumen by 0.5 to $1 \mathrm{~cm}$ and on the rostral side by at least $3 \mathrm{~cm}$ above the stricture (Illustration). Since 1992 we have placed 20 bilateral and 5 unilateral Wallstents to relieve obstruction by carcinoma of the cervix (10 Stage T3b \& 15 stage T4). Twenty of our patients received multiple chemotherapeutic cycles over 18 to 145 months. The technical successrate of wallstent placement was $100 \%$; 14 of the wallstents remained patent over 18 months and patients continued to have adequate renal function maintaining a creatinine level of $1.2-1.8[7]$.

However 56 endourologic procedures were necessary to assure continued patency [7]. Holmium laser ablation was used in 14 patients to manage tumor proliferation and ingrowth at the distal end of the wallstent [7]. In four patients the wall stents had to be extended to counter rostral tumor extension. Another 32 endo- stents and replacement stent were seated to assure continue patency [7].

In addition 11 wallstents were placed in patients with carcinoma of the prostate compromising the ureteric lumen, four with carcinoma of the rectosigmoid, 2 with endometrial carcinoma and two with carcinoma of the ovary. In these patients the wallstents remained patent for 4-92 months (mean 17 months). Four wallstents were placed in patients with retroperitoneal collaginosis compromising the ureter and in another 3 with endometriosis. The mean functional patency in this group was 42 months.

In the last 2 years we have replaced wallstents with thermoexpandable metallic stents (Memokath, PNM Medical, Denmark [8-11]).

\section{CONCLUSION}

Wallstents and thermoexpandable metallic ureteric stents offer a costeffective solution to the problem of restoring drainage in ureters compromised by encasing neoplasms, inflammatory ciccatricial changes, endometriosis and retroperitoneal collagenosis.

\section{ILLUSTRATION}

Two $10 \mathrm{~cm}$ wallstents in position, in patient with obstructing carcinoma of cervix. Note deviation of right wallstent by massive nodes.

\section{REFERENCES}

1. Kocot $A$, Kalogirou $C$, Vergho $D$, Riedmiller $H$. Long-term results of ileal ureteric replacement: a 25year single-center experience. BJU Int. 2017; 10: 13825. Ref.: https://goo.gl/wyvhM8

2. Wenske $\mathrm{S}$, Olsson $\mathrm{CA}$, Benson MC. Outcome of distal ureteral reconstruction through reimplantation with psoas hitch, Boari flap, or ureteroneocystostomy for benign or malignant ureteral obstruction or injury. Urology. 2013; 82: 231-236. Ref.: https://goo.gl/xurtWF

3. Gong $H, L u ~ Y$, Yan $Y$, Wu Z, Gao W, et al. Laparoscopic extravesical submucosal tunneling ureteroneocystostomy combined with psoas hitch for medium length distal ureteral defects in adults. Urol Int. 2017; 98: 343-349 Ref.: https://goo.gl/Mb8duF

4. Nicita G, Martini a, Filicamo MT, Saieva C, Tosto A, et al. Use of sigmoid colon in orthotopic neobladder reconstruction: Longterm results. Int J Urol. 2016; 23: 984-90. Ref.: https://goo.gl/KvtXKb 
5. Touiti D, Gelet A, Deligne E, Fassi FH, Fassi FH, et al. Treatment of uretero-intestinal and ureterovesical stenosis with the Acucise balloon catheter. Prog Urol. 2001; 11: 1224-1230. Ref.: https://goo.gl/10zHe5

6. Lang EK. Efficacy of Wallstents in treatment of ureteral obstruction caused by malignancies. European Radiology. 1997; 7: 31-32.

7. Lang EK, Winer AG, Abbey-Mensah G, Anne R, Allaei A, et al. Longterm results of metallic stents for malignant ureteral obstruction in advanced cervical carcinoma. J Endourol. 2013; 27: 646-651. Ref.: https://goo.gl/1VLPKO

8. EGEA Camacho J, Trueba Arquinarena J, Fernandez Dominguez, López Martín JJ, De La Cruz Ruiz $M$, et al. Self-expanding metallic endoprosthesis in the treatment of recurrent ureteral stricture. Arch Esp Urol. 1998; 51: 258-262 Ref.: https://goo.gl/YQnjbv

9. Patel C, Loughran D, Jones R, Abdulmajed M, Shergill I. The resonance R metallic ureteric stent in treatment of chronic ureteric obstruction: a safety and efficacy analysis from a contemporary clinical series. BMC Urol. 2017; 17: 16. Ref.: https://goo.gl/eF3Xqm

10. Kim M, Hong B, Park HK. Long-term outcomes of double-layered Polytetraflourethelene Membrane covered self-expandable segmental Metallic Stents (Uventa) in patients with chronic ureteral obstructions: Is it really safe? J. Endourol. 2016; 30: 1339-1346. Ref.: https://goo.gl/U0P7rB

11. Cox AC, Thomas JA. Insertion of thermoexpandable metallic ureteric stents can be aided by ureteric predilatation. Ann R Coll Surg Engl. 2016; 98: 158-159. Ref.: https://goo.gl/tB9Vo8 